\title{
Estudio observacional de los goles marcados y recibidos por la selección española en la Euro beach soccer 2016 de Sanxenxo y Moscú
}

\section{Observational study of the goals scored and received by the spanish national team in the Euro beach soccer 2016 in Sanxenxo and Moscow}

\author{
Luis Lois-Carro
}

'Real Federación española de Fútbol. Fútbol UEFA NACIONAL, Madrid, España. loiscarro@gmail.com

Cómo citar: Lois-Carro, L. 2022. Estudio observacional de los goles marcados y recibidos por la selección española en la Euro beach soccer 2016 de Sanxenxo y Moscú. Rev. Digit. Act. Fis. Deport. 8(1):e1876. http://doi.org/10.31910/rdafd.v8.n1.2022.1876

Artículo de acceso abierto publicado por Revista Digital: Actividad Física y Deporte, bajo una licencia Creative Commons CC BY-NC 4.0

Publicación oficial de la Universidad de Ciencias Aplicadas y Ambientales U.D.C.A, Institución de Educación Superior Acreditada de Alta Calidad por el Ministerio de Educación Nacional.

Recibido: agosto 16 de 2021 Aceptado: octubre 10 de 2021 Editado por: Álvaro José Gracia Díaz

\section{RESUMEN}

Introducción: El fútbol playa comenzó en Brasil, más concretamente en la playa Leme de Río de Janeiro y ha crecido para llegar a ser un deporte internacional. Objetivo: Analizar las acciones defensivas y ofensivas, que terminan en gol a favor y en contra de la selección española, en la Euro Beach Soccer League, que tuvo lugar en julio de 2016, en España y en Moscú. Los goles totales analizados fueron 47 y el número de partidos fue de 6 , destacando el interés que genera al analizar el equipo campeón, de esa edición. Metodología: Análisis observacional, mediante un instrumento de observación (LINCE) y un instrumento de registro Match Visión Studio Premium v.1.0. software. Los resultados, determinados mediante estadística descriptiva y análisis secuencial de T-Patterns, obtenido mediante el programa Theme 5.0 software. Resultados: Los resultados obtenidos muestran que las fases de ataque son, en su mayor parte, por medio de acciones combinativas. Conclusiones: La superioridad de los equipos que juegan en su propio país (Rusia y España) parece clara, quizás excesiva, respecto al resto de equipos que participan durante la Eurocopa, debido a que se ve incrementada la probabilidad que se consiga un número elevado de goles a favor ( $>4$ goles), en un partido.

Palabras clave: Fútbol playa; Análisis observacional; Selección Española; Goles.

\section{ABSTRACT}

Introduction: Beach soccer began in Brazil, more specifically on Leme Beach in Rio de Janeiro, and has grown to become an international sport. Objective: Analyze the defensive and offensive actions that end in goals for and against the Spanish national team in the Euro Beach Soccer League that took place in the month of July 2016 in Spain and Moscow. The total goals analyzed were 47 and the number of matches analyzed was 6 , highlighting the interest generated by analyzing the champion team of that edition. Methodology: For this purpose, an observational analysis was carried out using an observation instrument (LINCE) and a registration instrument Match Vision Studio Premium v.1.0. software. The results, determined by descriptive statistics and sequential analysis of T-Patterns obtained using Theme 5.0 software. Results: The results obtained 
show that the phases of attack in which success is in the majority by combinated action tactics. Conclusions: The superiority of the teams playing with the public in own country (Russia and Spain) seems clear, perhaps excessive, with respect to the other teams participating during the Eurocup since the probability of a high number of goals scored $(>4$ goals), in a match is increased.

Keywords: Beach soccer; Observational analysis; Spanish National Team; Goals.

\section{INTRODUCCIÓN}

El fútbol playa es una modalidad del fútbol que se juega sobre una superficie de arena lisa, entre dos equipos de cinco jugadores cada uno y cuyo objetivo es marcar más goles al equipo contrario. Este deporte tiene como objetivo el ocio y el espectáculo, más que la competitividad. Hay un tiro a puerta cada treinta segundos, un gol cada cuatro minutos y 16 equipos participan, en cada uno de los eventos. A excepción del país anfitrión, los 15 equipos restantes participan en un proceso clasificatorio dentro de cada una de las confederaciones continentales, las cuales, organizan diversos torneos (FIFA, 2015).

El fútbol playa comenzó en Brasil, más concretamente en la playa Leme en Río de Janeiro y ha crecido, para llegar a ser un deporte internacional. La participación de jugadores de fama internacional, como el francés Éric Cantona, legendarios delanteros españoles, como Míchel y Julio Salinas y estrellas brasileñas, como Romário, Júnior y Zico, han ayudado a ampliar la cobertura televisiva al público, de más de 170 países de todo el mundo, haciendo del fútbol playa, uno de los deportes profesionales con mayor crecimiento del mundo y la conversión en un espectáculo comercial internacional (FIFA, 2015).

A pesar que en el fútbol prima el espectáculo, al igual que cualquier deporte colectivo, el principal objetivo de su práctica es la consecución de un punto, gol o tanto y poder así ganar el encuentro que se está disputando. El ataque, como principal característica, debe superar los problemas de conservación individual y colectiva del balón, franquear, utilizar o evitar los obstáculos móviles, para llegar a marcar. Por ello, el objetivo de cada una de las acciones es provocar y explotar un desequilibrio en el esquema táctico contrario, creando un efecto sorpresa de incertidumbre para anotar el gol, en el caso del fútbol, fútbol sala y fútbol playa (Gréhaigne, 2001). Además, explican que las acciones anteriores al lanzamiento a portería mantienen una relación estable entre sí $y$, por consiguiente, pueden ser consideradas como excitadoras, para la consecución del éxito en el juego (Lago Peñas et al. 2003).

En consecuencia, la evaluación y el análisis de estas determinadas acciones durante una temporada completa es fundamental, para conocer cuáles son las variables o factores que pueden suponer el éxito deportivo. El estudio de estas prestaciones, tanto a nivel individual de jugadores como colectivo de equipos, constituye un aporte de información esencial para los entrenadores, que permite conocer las características de la actividad competitiva, en situaciones reales de juego (González et al. 2002).

Según Medina \& Delgado (1999), la observación es el procedimiento, mediante el cual, es posible obtener información de diversos acontecimientos o hechos, utilizando diferentes técnicas, que ayuden a la percepción del observador. Para el análisis de estas AO, que finalizan en gol, Medina \& Delgado (1999) y Anguera \& Hernández-Mendo (2013) proponen metodologías basadas en creación de diseños observacionales, según determinados criterios observables. Todo ello, siempre bajo un proceso de entrenamiento, que asegure la fiabilidad y evite distorsiones del estudio, incluyendo soportes informáticos o software, que ayuden a completar esta metodología.

Por lo tanto, el presente estudio tuvo como objeto analizar las acciones ofensivas y defensivas que terminan en gol, de la selección española de fútbol playa, durante la Euro BS League de Sanxenxo y Moscú, considerando que el fútbol playa es un deporte donde prima la espectacularidad y el juego colectivo, mediante la consecución de un número elevado de goles, la mayoría desde fuera del área y participando varios jugadores en la obtención de estos.

\section{MATERIALES Y MÉTODOS}

Se trata de un estudio observacional, descriptivo y comparativo, en el que se han analizado los goles que ha marcado y recibido la selección española de fútbol playa, en las dos jornadas de la liga europea, 


\section{Revista Digital: Actividad Física y Deporte}

que tuvieron lugar en julio, en Sanxenxo y Moscú. En total, se analizaron 47 goles.

Se ha utilizado la metodología observacional de Anguera (1999), que goza del necesario rigor y flexibilidad para estudiar los episodios que se presentan de forma natural en el proceso de análisis de los goles en fútbol playa. Basándonos en Heinemann (2003), se puede manifestar que el tipo de observación llevada a cabo ha sido estandarizada, abierta y no participante.

El presente estudio, se llevó a cabo por medio de una metodología observacional de Bakeman \& Gottman (1987). El diseño observacional, según Anguera (2003), se basa en un seguimiento de la disputa de todos los partidos jugados por la selección española, en la Euro liga, nomotético, ya que se estudia el número de veces que se repite un comportamiento, en este caso el gol y multidimensional, ya que trataremos de dar explicación a diferentes conductas que se lleva a cabo en un mismo tiempo, durante el gol, es decir, si el remate se produce con el empeine, interior de pie, exterior, de cabeza, tijera.
Por otro lado, si el remate se produce con el pie derecho o izquierdo; la zona del campo en la que se produce el remate dentro del área, entre el área y el medio del campo, el centro del campo y el propio campo; también, el tiempo de juego en el que se produce el remate.

El nivel de participación es una observación no participativa, dado que el observador no interactúa con los jugadores observados y el grado de perspicacia es la observación completa, directa.

Como se observa en la figura 1 , con relación al tiempo, se utilizó un parámetro de frecuencia y debido a que en este trabajo se estudia el gol, se analiza el número total de goles que marcó y recibió la selección española, en ambos torneos. Posteriormente, para facilitar su lectura, se expresó en porcentajes.

En total, se jugaron 24 encuentros entre todos los partidos de la liga europea, disputados en Sanxenxo y en Moscú; grabados y codificados, un total de seis encuentros.

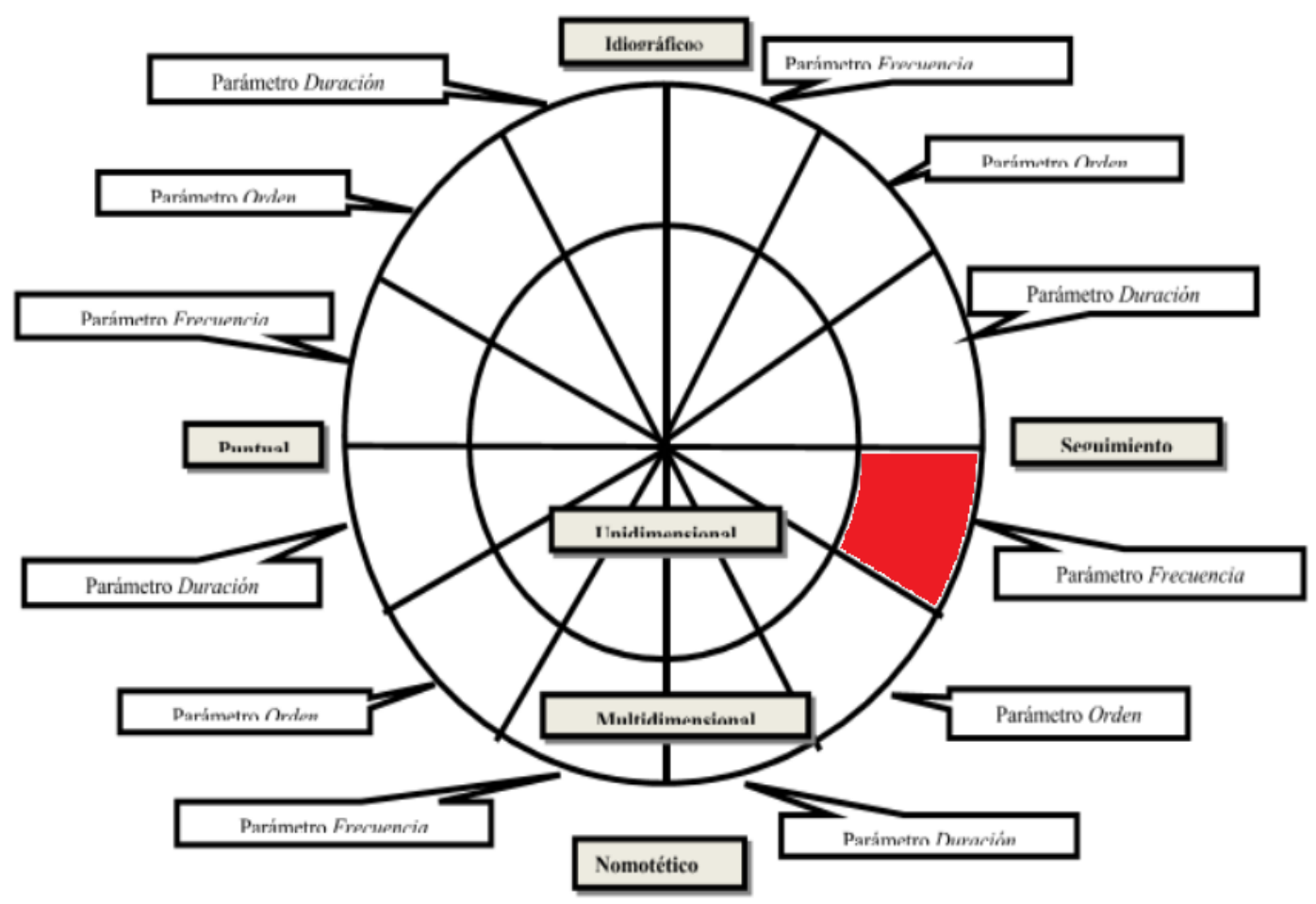

Figura 1. Registro de acciones para anotar y evitar el gol por parte de la Selección Española de fútbol playa para la Liga Europea y Liga Sanxenxo. 
Tabla 1. Tabla característica de los goles analizados.

\begin{tabular}{|c|c|c|c|c|}
\hline Campeonato & $\begin{array}{c}\text { Partidos } \\
\text { analizados }\end{array}$ & $\begin{array}{c}\text { Goles a } \\
\text { favor de } \\
\text { España }\end{array}$ & $\begin{array}{c}\text { Goles en } \\
\text { contra de } \\
\text { España }\end{array}$ & Goles totales \\
\hline $\begin{array}{c}\text { Liga } \\
\text { Europea } \\
\text { Sanxenxo }\end{array}$ & 3 & 22 & 6 & 28 \\
\hline $\begin{array}{c}\text { Liga } \\
\text { Europea } \\
\text { Moscú }\end{array}$ & 3 & 14 & 5 & 19 \\
\hline Total & 6 & 36 & 11 & 47 \\
\hline
\end{tabular}

El detalle de los campeonatos, partidos, goles a favor y en contra analizados en el presente estudio, se presentan en la tabla 1.

El presente estudio, se realizó mediante el Software y análisis de datos observacionales, Ilamado LINCE (multiplatform sport analysis software. Procedia-Social and Behavioral Sciences 46. 4692-4694), uno de los programas de registro más utilizados en metodología observacional. Las principales características de este Software es que es genérico, gratuito y flexible, permite la correcta visualización y registro de acciones de juego en video; gracias al análisis Kappa, se puede obtener un control adecuado de la calidad de dato, el análisis es en tiempo real y el programa permite un análisis del dato, en el que se pueden extraer los datos, a diferentes hojas de cálculo.

También, se utilizó la distribución de Poisson y la distribución binomial negativa, para el análisis de los goles de la selección española.

En este estudio, se analizaron los goles desde diferentes perspectivas, teniendo en cuenta diferentes ítems, para poder lograr un mayor éxito, rigor y fiabilidad en la investigación. El protocolo y la metodología que se llevó a cabo fue la establecida por Anguera \& Hernández-Mendo (2013), para este tipo de estudios observacionales, que no es otra que el diseño observacional.

\section{RESULTADOS Y DISCUSIÓN}

Relación entre la zona de golpeo y la consecución de un gol. Como se mencionó, el fútbol playa es un deporte donde se observan un gran número de goles en cada partido, donde prima la espectacularidad, lo cual, se corroboró en el presente estudio (Tabla 2), ya que la mayoría de los goles, se marcaron desde fuera del área Y Z3; sin embargo, los goles desde dentro del área, también son habituales. En concreto, desde dentro del área, se marca un 44,68 \% de los goles; le sigue la Z2, con un $40,25 \%$ y el Z3, con $15,89 \%$. Si se juntan ambos porcentajes anteriores, se puede observar que el 56,14 \% de los goles del torneo, se marcaron desde fuera del área. También, se debe señalar, que no se marcó ningún gol desde el campo propio, por lo que también se puede afirmar que no es una acción de ataque productiva.

Tabla 2. Análisis de los goles de acuerdo con la zona de golpeo, superficie de golpeo y pie de golpeo.

\begin{tabular}{|c|c|c|c|c|c|c|}
\hline \multicolumn{7}{|c|}{ Zona de golpeo } \\
\hline \multirow{2}{*}{ Zona de golpeo } & \multicolumn{3}{|c|}{$\begin{array}{c}\text { Número de goles } \\
\text { marcados }\end{array}$} & \multicolumn{3}{|c|}{$\%$ de goles marcados } \\
\hline & $\begin{array}{l}\text { Sanxenxo } \\
\text { (28) }\end{array}$ & $\begin{array}{l}\text { Moscú } \\
\text { (19) }\end{array}$ & $\begin{array}{l}\text { Total } \\
(47)\end{array}$ & $\begin{array}{l}\text { Sanxenxo } \\
\%\end{array}$ & $\begin{array}{c}\text { Moscú } \\
\%\end{array}$ & $\begin{array}{l}\text { Total } \\
\%\end{array}$ \\
\hline $\mathrm{Z1}$ & 9 & 12 & 21 & 32,15 & 63,17 & 44,68 \\
\hline Z2 & 14 & 5 & 19 & 50,00 & 26,31 & 40,25 \\
\hline Z3 & 5 & 2 & 7 & 17,85 & 10,52 & 15,89 \\
\hline Z4 & 0 & 0 & 0 & 0 & 0 & 0 \\
\hline
\end{tabular}


Continuación tabla 2

\begin{tabular}{|c|c|c|c|c|c|c|}
\hline \multicolumn{7}{|c|}{ Superficie de golpeo } \\
\hline \multirow{2}{*}{$\begin{array}{l}\text { Superficie de } \\
\text { golpeo }\end{array}$} & \multicolumn{3}{|c|}{$\begin{array}{l}\text { Número de goles } \\
\text { marcados }\end{array}$} & \multicolumn{3}{|c|}{$\%$ de goles marcados } \\
\hline & $\begin{array}{l}\text { Sanxenxo } \\
(28)\end{array}$ & $\begin{array}{l}\text { Moscú } \\
\text { (19) }\end{array}$ & $\begin{array}{l}\text { Total } \\
(47)\end{array}$ & $\begin{array}{c}\text { Sanxenxo } \\
\%\end{array}$ & $\begin{array}{c}\text { Moscú } \\
\%\end{array}$ & $\begin{array}{c}\text { Total } \\
\%\end{array}$ \\
\hline $\mathrm{CA}$ & 0 & 3 & 3 & 0 & 15,78 & 6,38 \\
\hline EM & 20 & 9 & 29 & 71,42 & 47,36 & 61,70 \\
\hline $\mathrm{IP}$ & 3 & 4 & 7 & 10,71 & 21,05 & 14,89 \\
\hline EP & 0 & 0 & 0 & 0 & 0 & 0 \\
\hline TJ & 3 & 1 & 4 & 10,71 & 5,26 & 8,51 \\
\hline PP & 1 & 1 & 2 & 3,57 & 5,26 & 4,25 \\
\hline OT & 1 & 1 & 2 & 3,57 & 5,26 & 4,25 \\
\hline \multicolumn{7}{|c|}{ Pie de golpeo } \\
\hline \multirow{2}{*}{ Pie de golpeo } & \multicolumn{3}{|c|}{$\begin{array}{c}\text { Número de goles } \\
\text { marcados }\end{array}$} & \multicolumn{3}{|c|}{$\%$ de goles marcados } \\
\hline & $\begin{array}{l}\text { Sanxenxo } \\
\text { (28) }\end{array}$ & $\begin{array}{l}\text { Moscú } \\
\text { (19) }\end{array}$ & $\begin{array}{c}\text { Total } \\
(47)\end{array}$ & $\begin{array}{c}\text { Sanxenxo } \\
\%\end{array}$ & $\begin{array}{c}\text { Moscú } \\
\%\end{array}$ & $\begin{array}{c}\text { Total } \\
\%\end{array}$ \\
\hline Pie derecho & 17 & 8 & 25 & 60,71 & 42,10 & 53,19 \\
\hline Pie izquierdo & 10 & 8 & 18 & 35,71 & 36,84 & 38,29 \\
\hline Oras partes & 1 & 3 & 4 & 3,57 & 15,78 & 8,55 \\
\hline
\end{tabular}

Relación entre la superficie de golpeo y la consecución de un gol. La mayoría de los goles obtenidos durante el torneo fueron con el pie, concretamente, con el empeine y con el interior del pie (Tabla 2). De acuerdo con las reglas de juego de fútbol playa, las tijeras son una acción habitual en este deporte y el árbitro debe protegerlas. Se trata de una filigrana llamativa propia de un deporte, donde prima la espectacularidad, por ello cabe destacar el porcentaje tan alto que tiene en la consecución de goles, en concreto, con un 8,51 \%, un porcentaje que no es habitual en el fútbol y fútbol playa.
Relación entre el tipo de golpeo y la consecución de un gol. La gran mayoría de los goles son obtenidos a media altura (Tabla 3), esto podría tener una explicación lógica, que no es otra que muchos tiros que van rasos acaban entrando a media altura, con motivo del bote inesperado que produce la superficie de juego (arena fina libre de conchas y guijarros), en el lanzamiento del balón.

Tabla 3. Análisis de los goles de acuerdo con el tipo de golpeo, tipo de jugada y número de jugadores que intervienen.

\begin{tabular}{|c|c|c|c|c|c|c|}
\hline \multicolumn{7}{|c|}{ Tipo de golpeo } \\
\hline \multirow{2}{*}{ Tipo de golpeo } & \multicolumn{3}{|c|}{$\begin{array}{c}\text { Número de goles } \\
\text { marcados }\end{array}$} & \multicolumn{3}{|c|}{$\%$ de goles marcados } \\
\hline & $\begin{array}{l}\text { Sanxenxo } \\
\text { (28) }\end{array}$ & $\begin{array}{l}\text { Moscú } \\
\text { (19) }\end{array}$ & $\begin{array}{l}\text { Total } \\
(47)\end{array}$ & $\begin{array}{c}\text { Sanxenxo } \\
\%\end{array}$ & $\begin{array}{c}\text { Moscú } \\
\%\end{array}$ & $\begin{array}{c}\text { Total } \\
\%\end{array}$ \\
\hline Alto & 7 & 4 & 11 & 25 & 21,05 & 23,40 \\
\hline Media altura & 12 & 10 & 22 & 42,85 & 52,63 & 46,80 \\
\hline Raso & 9 & 5 & 14 & 32,14 & 26,31 & 29,78 \\
\hline \multicolumn{7}{|c|}{ Tipo de jugada } \\
\hline \multirow{2}{*}{ Tipo de jugada } & \multicolumn{3}{|c|}{$\begin{array}{c}\text { Número de goles } \\
\text { marcados }\end{array}$} & \multicolumn{3}{|c|}{$\%$ de goles marcados } \\
\hline & $\begin{array}{l}\text { Sanxenxo } \\
(28)\end{array}$ & $\begin{array}{l}\text { Moscú } \\
\text { (19) }\end{array}$ & $\begin{array}{l}\text { Total } \\
(47)\end{array}$ & $\begin{array}{c}\text { Sanxenxo } \\
\%\end{array}$ & $\begin{array}{c}\text { Moscú } \\
\%\end{array}$ & $\begin{array}{c}\text { Total } \\
\%\end{array}$ \\
\hline Individual & 15 & 7 & 22 & 53,57 & 36,84 & 46,80 \\
\hline Colectiva & 13 & 12 & 25 & 46,42 & 63,15 & 53,19 \\
\hline
\end{tabular}


Continuación tabla 3

\begin{tabular}{|c|c|c|c|c|c|c|}
\hline \multicolumn{7}{|c|}{ Número de jugadores que intervienen } \\
\hline \multirow{2}{*}{$\begin{array}{l}\text { Número de } \\
\text { jugadores que } \\
\text { intervienen }\end{array}$} & \multicolumn{3}{|c|}{$\begin{array}{c}\text { Número de goles } \\
\text { marcados }\end{array}$} & \multicolumn{3}{|c|}{$\%$ de goles marcados } \\
\hline & $\begin{array}{l}\text { Sanxenxo } \\
(28)\end{array}$ & $\begin{array}{l}\text { Moscú } \\
\text { (19) }\end{array}$ & $\begin{array}{l}\text { Total } \\
(47)\end{array}$ & $\begin{array}{l}\text { Sanxenxo } \\
\%\end{array}$ & $\begin{array}{l}\text { Moscú } \\
\%\end{array}$ & $\begin{array}{l}\text { Total } \\
\%\end{array}$ \\
\hline 1 jugador & 15 & 8 & 23 & 53,57 & 42,10 & 48,93 \\
\hline 2 jugadores & 11 & 7 & 18 & 39,28 & 36,84 & 38,29 \\
\hline 3 jugadores & 2 & 4 & 6 & 7,14 & 21,05 & 12,76 \\
\hline
\end{tabular}

Relación entre el juego individual-colectivo y la consecución de un gol. El fútbol playa es un deporte donde prima el juego colectivo. Debido a la dificultad que se tiene para avanzar con el balón, por la superficie de arena fina, los jugadores se ven obligados a ir pasándose el balón hasta encontrarse en una zona próxima a la portería y tirar a gol. Debido a esta situación, no destaca que un 53,19 \% de los goles, se hayan conseguido por medio de acciones colectivas (Tabla 3).

Relación entre el estado del partido y la consecución de un gol. De acuerdo con las reglas de juego de fútbol playa, en la regla 5, el reglamento imprime que la ley de la ventaja debe ser en ocasiones claras de gol, ya que el lanzamiento de un tiro libre sin barrera suele ser aprovechadas eficazmente por los jugadores. Muestra de ello, es que el 34,04\% de los goles son obtenidos con lanzamientos de tiro libre (Tabla 4). Teniendo en cuenta esta situación, se observa la importancia que tiene el entrenamiento de la táctica. Este hecho, también tiene relación con el apartado anterior, ya que los lanzamientos directos a portería son contabilizados como goles individuales.

Tabla 4. Análisis de los goles, de acuerdo con el estado del partido y al tiempo de juego.

\begin{tabular}{|c|c|c|c|c|c|c|}
\hline \multicolumn{7}{|c|}{ Estado del partido } \\
\hline \multirow{2}{*}{$\begin{array}{l}\text { Estado del } \\
\text { partido }\end{array}$} & \multicolumn{3}{|c|}{\begin{tabular}{|c|}
$\begin{array}{c}\text { Número de goles } \\
\text { marcados }\end{array}$ \\
\end{tabular}} & \multicolumn{3}{|c|}{$\%$ de goles marcados } \\
\hline & $\begin{array}{l}\text { Sanxenxo } \\
(28)\end{array}$ & $\begin{array}{l}\text { Moscú } \\
\text { (19) }\end{array}$ & $\begin{array}{l}\text { Total } \\
\text { (47) }\end{array}$ & $\begin{array}{c}\text { Sanxenxo } \\
\%\end{array}$ & $\begin{array}{c}\text { Moscú } \\
\%\end{array}$ & $\begin{array}{c}\text { Total } \\
\%\end{array}$ \\
\hline Activo & 18 & 13 & 31 & 64,28 & 68,42 & 65,95 \\
\hline Pasivo & 10 & 6 & 16 & 35,71 & 31,57 & 34,04 \\
\hline \multicolumn{7}{|c|}{ Tiempo de juego } \\
\hline \multirow{2}{*}{ Tiempo de juego } & \multicolumn{3}{|c|}{$\begin{array}{l}\text { Número de goles } \\
\text { marcados }\end{array}$} & \multicolumn{3}{|c|}{$\%$ de goles marcados } \\
\hline & $\begin{array}{c}\text { Sanxenxo } \\
(28)\end{array}$ & $\begin{array}{l}\text { Moscú } \\
\text { (19) }\end{array}$ & $\begin{array}{c}\text { Total } \\
(47)\end{array}$ & $\begin{array}{c}\text { Sanxenxo } \\
\%\end{array}$ & $\begin{array}{c}\text { Moscú } \\
\%\end{array}$ & $\begin{array}{c}\text { Total } \\
\%\end{array}$ \\
\hline $1^{\circ}$ tiempo & 15 & 8 & 23 & 53,57 & 42,10 & 48,93 \\
\hline $2^{\circ}$ tiempo & 6 & 7 & 13 & 21,42 & 36,84 & 27,65 \\
\hline $3^{\circ}$ tiempo & 7 & 4 & 11 & 32,14 & 21,05 & 23,40 \\
\hline
\end{tabular}

Relación entre los tiempos de juego y la consecución de un gol. El jugar en una superficie de arena fina provoca un gran desgaste físico en los jugadores; ésto, también tiene repercusión en la consecución de los goles. Cuanta más energía y más frescos están los jugadores más posibilidades tienen de conseguir gol; por ello, se destaca que un 48,93\% de los goles son conseguidos en el primer tiempo (Tabla 4).
Relación entre la influencia del público y la consecución de un gol. El público siempre genera un gran interés en los analistas e investigadores deportivos. En este estudio, también se analizó la diferencia de goles marcados por la selección española con el apoyo del público y sin su apoyo. La diferencia de gol marcado en cada torneo ha sido muy representativa, debido a que, en Sanxenxo, con alrededor de 2.500 personas 
en el estadio, la gran mayoría, con constante apoyo hacia la selección española en cada partido, tuvo efecto en los jugadores, por lo que han logrado un total de 22 goles en relación a los 14 goles que marcó la selección en Moscú, es decir, España logró 2,6 goles más por partido en Sanxenxo, con un gran apoyo del público, que en Moscú, donde se encontraba en un terreno hostil (Tabla 5).

Tabla 5. Análisis de los goles, de acuerdo con el campeonato de goles marcados-recibidos.

\begin{tabular}{|c|c|c|c|c|c|c|}
\hline \multirow{2}{*}{ Goles } & \multicolumn{3}{|c|}{$\begin{array}{c}\text { Número de goles } \\
\text { marcados }\end{array}$} & \multicolumn{2}{c|}{$\%$ de goles marcados } \\
\cline { 2 - 7 } & $\begin{array}{c}\text { Sanxenxo } \\
(\mathbf{2 8 )}\end{array}$ & $\begin{array}{c}\text { Moscú } \\
(\mathbf{1 9})\end{array}$ & $\begin{array}{c}\text { Total } \\
\mathbf{( 4 7 )}\end{array}$ & $\begin{array}{c}\text { Sanxenxo } \\
\%\end{array}$ & $\begin{array}{c}\text { Moscú } \\
\%\end{array}$ & $\begin{array}{c}\text { Total } \\
\%\end{array}$ \\
\hline Marcados & 22 & 14 & 36 & 78,57 & 73,68 & 76,59 \\
\hline Recibidos & 6 & 5 & 11 & 21,42 & 26,31 & 34,04 \\
\hline
\end{tabular}

Calidad de los datos analizados. El control de la calidad del dato, se ha llevado a cabo mediante concordancia intra observador. En concreto, la fiabilidad en forma de concordancia, se ha calculado de manera cuantitativa, mediante el coeficiente Kappa de Cohen $(1960,1966)$, ya que se trata de una medida de concordancia para clasificaciones nominales, en las que no existe un orden de graduación entre las diferentes categorías. El cálculo del índice Kappa de Cohen, se realizó con el programa informático LINCE vL.2., teniendo en consideración las recomendaciones de Bakeman et al. (1996) y de Bakeman \& Quera (2001). Se obtuvo un valor del Kappa del conjunto $(0,96)$, que supone, a partir de Landis \& Koch (1977), una consideración del acuerdo: "almost perfect".

\section{CONCLUSIONES}

Una vez finalizado el análisis estadístico descriptivo y el análisis observacional de los goles marcados y recibidos por la selección española y tras un proceso de interpretación y discusión de este, las conclusiones de la presente investigación son las siguientes:

El empeine es el recurso técnico más utilizado para tirar a portería y obtener gol y la mayoría de los goles, se obtienen desde fuera del área. El pie más utilizado para obtener gol es el derecho.

Las jugadas a balón parado tienen una gran importancia para poder conseguir un gol, ya que estos lanzamientos son directamente a portería y sin barrera.

El fútbol playa es un deporte donde destaca el ataque colaborativo, debido a la gran dificultad que tiene regatear o correr con el balón por la arena fina, por lo que el juego colectivo es una premisa fundamental, en este deporte.

La presencia de un público hostil o no puede tener influencia en la consecución de los goles durante el partido, ya que España ha conseguido marcar más goles en el campeonato de Sanxenxo que en el campeonato de Moscú.

Conflictos de intereses: El manuscrito fue preparado y revisado con la participación de todos los autores, quienes declaramos que no existe ningún conflicto de intereses que ponga en riesgo la validez de los resultados presentados.

\section{REFERENCIAS}

1. ANGUERA, M. 2003. Observational Methods (General). In: Fernández-Ballesteros, R. (Ed.), Encyclopedia of psychological assessment. 2:632-637.

2. ANGUERA, M.; HERNÁNDEZ-MENDO, A. 2013. The observational methodology in the field of sport. Journal of Sport Sciences. 9:135-160.

3. ANGUERA, M.T. 1999. Hacia una evaluación de la actividad cotidiana y su contexto: ¿presente o futuro para la metodología? Discurso de ingreso como académica numeraria electa. Barcelona: Real Academia de Doctores.

4. BAKEMAN, R.; QUERA, V. 2001. Using GSEQ with SPSS. Metodología de las Ciencias del Comportamiento. 3(2):195-214 
5. BAKEMAN, R.; MCARTHUR, D.; QUERA, V. 1996. Detecting group differences in sequential association using sampled permutations: Log odds, kappa, and phi compared. Behavior Research Methods, Instruments and Computers. 28(3):446-457. https://doi.org/10.3758/BF03200524

6. BAKEMAN, R.; GOTTMAN, J. 1987. Applying observational methods: A systematic view. In: Osofsky, J.D. (Ed.), Handbook of infant development. 2nd edition, Wiley. New York, E.E.U.U. p. 818-853.

7. FEDERACIÓN INTERNACIONAL DE FÚTBOL ASOCIACIÓN, FIFA. 2015. Historia del beach soccer. FIFA. Disponible desde Internet en: http://es.fifa.com/beachsoccerworldcup/ organisation/history/index.html

8. GONZÁLEZ, C.; UREÑA, A.; SANTOS, A.; LLOP, F. NAVARRO, F. 2002. The Libero analysis of the characteristics of their game in the volleyball competition. Revista de Motricidad. 8:141-159.
9. GRÉHAIGNE, J. 2001. The organization of the game in football. INDE. Barcelona, España.

10. HEINEMANN, K. 2003. Introducción a la metodología de la investigación empírica. Paidotribo. Barcelona, España. p.285

11. LAGO PEÑAS, C.; CANCELA CARRAL, J.M.; FERNÁNDEZ FRAGA, F.; LÓPEZ GRAÑA, M. DEL P.; VEIGA CODESIDO, J. 2003. Evaluation of offensive actions in football performance against indicators of success in intensive diachronic retrospective designs. Apunts: Educación física y deportes. 72:96-103.

12. LANDIS, J.; KOCH, G. 1977. The measurement of observer agreement for categorial data. Biometrics. 33:159-174.

https://doi.org/10.2307/2529310

13. MEDINA, J.; DELGADO, M. 1999. Methodology training of observers to researchers EF and Sports in which is used as a method observation. Revista de Motricidad. 5:69-86. 